\title{
CORRIGENDUM
}

\section{Corrigendum to "Upper water structure and mixed layer depth in tropical waters: The SEATS station in the Northern South China Sea"}

\section{[Terr. Atmos. Ocean. Sci., Vol. 28, No. 6, 1019-1032, December 2017]}

\author{
Jen-Hua Tai ${ }^{1, *}$, George T. F. Wong ${ }^{1,2}$, and Xiaoju Pan ${ }^{3}$ \\ ${ }^{1}$ Research Center for Environmental Changes, Academia Sinica, Taipei City, Taiwan \\ ${ }^{2}$ Department of Ocean, Earth and Atmospheric Sciences, Old Dominion University, Norfolk, VA \\ ${ }^{3}$ Key Laboratory of Marine Chemistry Theory and Technology, Ministry of Education, Ocean University of China, Qingdao, \\ China
}

Received 6 October 2020, revised 6 November 2020, accepted 8 November 2020

Recently, we found that the table captions of Tai et al. (2017) were not detailed enough. To make it easy for readers to understand the content of the tables, its captions have been corrected as shown below.

Table 1. Statistics of station occupations at the SEATS station and its nearby waters.

\begin{tabular}{|c|c|c|c|c|c|c|c|c|c|c|c|c|c|}
\hline Year & Jan & Feb & Mar & Apr & May & Jun & Jul & Aug & Sep & Oct & Nov & Dec & $\begin{array}{c}\text { Annual total } \\
\mathbf{N}(\mathbf{A} / \mathbf{B})\end{array}$ \\
\hline 1997 & 0 & 0 & 0 & $1(0 / 1)$ & 0 & $1(0 / 1)$ & 0 & 0 & 0 & $1(0 / 1)$ & 0 & 0 & $3(0 / 3)$ \\
\hline 1998 & 0 & 0 & 0 & 0 & $1(0 / 1)$ & $1(0 / 1)$ & 0 & 0 & 0 & 0 & 0 & 0 & $2(0 / 2)$ \\
\hline 1999 & 0 & 0 & 0 & $1(0 / 1)$ & 0 & 0 & 0 & $1(0 / 1)$ & $2(1 / 1)$ & 0 & $1(1 / 0)$ & $1(0 / 1)$ & $6(2 / 4)$ \\
\hline 2000 & $1(1 / 0)$ & 0 & $2(1 / 1)$ & 0 & $1(1 / 0)$ & 0 & $2(2 / 0)$ & 0 & 0 & $1(1 / 0)$ & 0 & 0 & $7(6 / 1)$ \\
\hline 2001 & 0 & $1(1 / 0)$ & $1(0 / 1)$ & 0 & 0 & $1(1 / 0)$ & 0 & 0 & 0 & $2(1 / 1)$ & 0 & $1(1 / 0)$ & $6(4 / 2)$ \\
\hline 2002 & 0 & 0 & $2(1 / 1)$ & 0 & 0 & 0 & $1(1 / 0)$ & 0 & $1(1 / 0)$ & $1(1 / 0)$ & $1(1 / 0)$ & 0 & $6(5 / 1)$ \\
\hline 2003 & $1(1 / 0)$ & 0 & $1(1 / 0)$ & 0 & 0 & 0 & $1(0 / 1)$ & $1(1 / 0)$ & 0 & $1(1 / 0)$ & 0 & $1(1 / 0)$ & $6(5 / 1)$ \\
\hline 2004 & 0 & 0 & $1(1 / 0)$ & 0 & $1(1 / 0)$ & $1(0 / 1)$ & 0 & $1(1 / 0)$ & 0 & 0 & $1(1 / 0)$ & 0 & $5(4 / 1)$ \\
\hline 2005 & $1(1 / 0)$ & 0 & $1(1 / 0)$ & 0 & 0 & 0 & $1(1 / 0)$ & 0 & 0 & 0 & $1(1 / 0)$ & $1(1 / 0)$ & $5(5 / 0)$ \\
\hline 2006 & 0 & 0 & 0 & 0 & 0 & $1(0 / 1)$ & $2(1 / 1)$ & $1(0 / 1)$ & 0 & $1(1 / 0)$ & 0 & 0 & $5(2 / 3)$ \\
\hline 2007 & $2(2 / 0)$ & 0 & 0 & 0 & 0 & 0 & $1(1 / 0)$ & 0 & 0 & $1(1 / 0)$ & 0 & 0 & $4(4 / 0)$ \\
\hline 2008 & 0 & 0 & 0 & 0 & $1(0 / 1)$ & 0 & 0 & 0 & 0 & 0 & 0 & $1(0 / 1)$ & $2(0 / 2)$ \\
\hline 2009 & 0 & 0 & 0 & 0 & 0 & 0 & $1(0 / 1)$ & 0 & 0 & 0 & 0 & 0 & $1(0 / 1)$ \\
\hline 2010 & 0 & 0 & 0 & 0 & 0 & 0 & 0 & 0 & 0 & $1(1 / 0)$ & 0 & 0 & $1(1 / 0)$ \\
\hline 2011 & 0 & 0 & 0 & 0 & 0 & 0 & 0 & 0 & 0 & 0 & 0 & $1(1 / 0)$ & $1(1 / 0)$ \\
\hline 2012 & 0 & 0 & 0 & 0 & 0 & 0 & 0 & $1(1 / 0)$ & 0 & 0 & 0 & 0 & $1(1 / 0)$ \\
\hline 2013 & 0 & 0 & 0 & $1(1 / 0)$ & 0 & 0 & 0 & 0 & 0 & $1(1 / 0)$ & 0 & $1(1 / 0)$ & $3(3 / 0)$ \\
\hline \multirow[b]{2}{*}{ Total } & $5(5 / 0)$ & $1(1 / 0)$ & $8(5 / 3)$ & $3(1 / 2)$ & $4(2 / 2)$ & $5(1 / 4)$ & $9(6 / 3)$ & $5(3 / 2)$ & $3(2 / 1)$ & $10(8 / 2)$ & $4(4 / 0)$ & $7(5 / 2)$ & $64(43 / 21)$ \\
\hline & \multicolumn{4}{|c|}{$\begin{array}{l}\text { Northeast monsoon } \\
\text { (Nov - Apr): } 28(21 / 7)\end{array}$} & $\begin{array}{c}\text { I (May) } \\
4(2 / 2)\end{array}$ & \multicolumn{4}{|c|}{$\begin{array}{l}\text { Southwest monsoon } \\
\text { (Jun - Sep): } 22(12 / 10)\end{array}$} & $\begin{array}{l}\mathrm{I}(\mathrm{Oct}) \\
10(8 / 2)\end{array}$ & \multicolumn{2}{|c|}{$\begin{array}{l}\text { Northeast monsoon } \\
\text { (Nov - Apr): } 28(21 / 7)\end{array}$} & \\
\hline
\end{tabular}

Note: N(A/B): N-Number of occupations; A - Occupations at SEATS station; B - occupations in vicinity of SEATS station; I-Inter-monsoonal period.

\footnotetext{
* Corresponding author

E-mail:jhtai@gate.sinica.edu.tw
} 
Table 2. Statistics of the types of hydrographic structures in the mixed layer.

\begin{tabular}{|c|c|c|c|c|c|c|c|c|c|c|c|c|c|c|c|}
\hline \multirow{2}{*}{ Year } & \multirow{2}{*}{ Jan } & \multirow{2}{*}{ Feb } & \multirow{2}{*}{ Mar } & \multirow{2}{*}{ Apr } & \multirow{2}{*}{ May } & \multirow{2}{*}{ Jun } & \multirow{2}{*}{ Jul } & \multirow{2}{*}{ Aug } & \multirow{2}{*}{ Sep } & \multirow{2}{*}{ Oct } & \multirow{2}{*}{ Nov } & \multirow{2}{*}{ Dec } & \multicolumn{3}{|c|}{ Annual Type Total } \\
\hline & & & & & & & & & & & & & (a) & (b) & (c) \\
\hline 1997 & - & - & - & $\mathrm{c}$ & - & $\mathrm{a}$ & - & - & - & $\mathrm{a}$ & - & - & 2 & 0 & 1 \\
\hline 1998 & - & - & - & - & $\mathrm{c}$ & $\mathrm{a}$ & - & - & - & - & - & - & 1 & 0 & 1 \\
\hline 1999 & - & - & - & $\mathrm{a}$ & - & - & - & $\mathrm{b}$ & $\mathrm{a}, \mathrm{a}$ & - & $\mathrm{a}$ & $\mathrm{a}$ & 5 & 1 & 0 \\
\hline 2000 & $\mathrm{~b}$ & - & $\mathrm{b}, \mathrm{c}$ & - & $\mathrm{a}$ & - & $\mathrm{a}, \mathrm{b}$ & - & - & $\mathrm{a}$ & - & - & 3 & 3 & 1 \\
\hline 2001 & - & $\mathrm{a}$ & $\mathrm{c}$ & - & - & $\mathrm{a}$ & - & - & - & $\mathrm{a}, \mathrm{a}$ & - & $\mathrm{a}$ & 5 & 0 & 1 \\
\hline 2002 & - & - & $\mathrm{a}, \mathrm{a}$ & - & - & - & $\mathrm{c}$ & - & $\mathrm{a}$ & $\mathrm{a}$ & $\mathrm{a}$ & - & 5 & 0 & 1 \\
\hline 2003 & $\mathrm{~b}$ & - & $\mathrm{c}$ & - & - & - & $\mathrm{a}$ & $\mathrm{c}$ & - & $\mathrm{a}$ & - & $\mathrm{a}$ & 3 & 1 & 2 \\
\hline 2004 & - & - & $\mathrm{c}$ & - & $\mathrm{a}$ & $\mathrm{a}$ & - & $\mathrm{a}$ & - & - & $\mathrm{a}$ & - & 4 & 0 & 1 \\
\hline 2005 & $\mathrm{a}$ & - & $\mathrm{a}$ & - & - & - & $\mathrm{a}$ & - & - & - & $\mathrm{b}$ & $\mathrm{a}$ & 4 & 1 & 0 \\
\hline 2006 & - & - & - & - & - & $\mathrm{a}$ & $\mathrm{a}, \mathrm{a}$ & $\mathrm{a}$ & - & $\mathrm{a}$ & - & - & 5 & 0 & 0 \\
\hline 2007 & $\mathrm{a}, \mathrm{c}$ & - & - & - & - & - & $\mathrm{a}$ & - & - & $\mathrm{a}$ & - & - & 3 & 0 & 1 \\
\hline 2008 & - & - & - & - & $\mathrm{a}$ & - & - & - & - & - & - & $\mathrm{a}$ & 2 & 0 & 0 \\
\hline 2009 & - & - & - & - & - & - & $\mathrm{a}$ & - & - & - & - & - & 1 & 0 & 0 \\
\hline 2010 & - & - & - & - & - & - & - & - & - & $\mathrm{a}$ & - & - & 1 & 0 & 0 \\
\hline 2011 & - & - & - & - & - & - & - & - & - & - & - & $\mathrm{a}$ & 1 & 0 & 0 \\
\hline 2012 & - & - & - & - & - & - & - & $\mathrm{a}$ & - & - & - & - & 1 & 0 & 0 \\
\hline 2013 & - & - & - & $\mathrm{c}$ & - & - & - & - & - & $\mathrm{a}$ & - & $\mathrm{a}$ & 2 & 0 & 1 \\
\hline \multicolumn{13}{|c|}{ Monthly Type Total } & \multicolumn{3}{|c|}{ Type Total } \\
\hline (a) & 2 & 1 & 3 & 1 & 3 & 5 & 7 & 3 & 3 & 10 & 3 & 7 & $48(75 \%)$ & & \\
\hline (b) & 2 & 0 & 1 & 0 & 0 & 0 & 1 & 1 & 0 & 0 & 1 & 0 & & $6(10 \%)$ & \\
\hline \multirow[t]{2}{*}{ (c) } & 1 & 0 & 4 & 2 & 1 & 0 & 1 & 1 & 0 & 0 & 0 & 0 & & & $10(15 \%)$ \\
\hline & \multicolumn{4}{|c|}{ Northeast monsoon } & I & \multicolumn{4}{|c|}{ Southwest monsoon } & I & \multicolumn{2}{|c|}{$\begin{array}{l}\text { Northeast } \\
\text { Monsoon }\end{array}$} & & & \\
\hline
\end{tabular}

Note: $a$-Classical type; $b$-Stepwise type; $c$ - Graded type; I - Internal-monsoonal period.

Table 3. Results in model II linear regression analyses relative to $\mathrm{MLD}_{\mathrm{T}}$.

\begin{tabular}{|c|c|c|c|c|c|c|c|c|c|c|}
\hline Type & Statistical Parameter & MLD $_{\mathrm{T} 2}$ & MLD $_{\mathrm{T} 5}$ & MLD $_{\mathrm{T} 10}$ & MLD $_{\theta G}$ & MLD $_{\sigma}$ & MLD $_{\sigma \mathrm{G} 1}$ & MLD $^{*}{ }_{\sigma \mathrm{G} 5}$ & MLD $^{*}{ }_{\sigma \mathrm{G} 10}$ & MLD $_{\text {mdg }}$ \\
\hline \multirow{10}{*}{ Classical } & A & -5 & -2 & 1 & -3 & -2 & -6 & -3 & -1 & 3 \\
\hline & B & 0.96 & 1.01 & 1.00 & 0.98 & 0.94 & 0.98 & 1.01 & 1.04 & 1.02 \\
\hline & $\sigma_{\mathrm{A}}$ & 3 & 1 & 1 & 1 & 2 & 2 & 2 & 2 & 2 \\
\hline & $\sigma_{\mathrm{B}}$ & 0.05 & 0.02 & 0.01 & 0.03 & 0.04 & 0.04 & 0.03 & 0.04 & 0.04 \\
\hline & $\gamma$ & 0.92 & 0.99 & 1.00 & 0.98 & 0.96 & 0.96 & 0.98 & 0.97 & 0.96 \\
\hline & $\operatorname{RMSE}_{1}(\mathrm{~m})$ & 11 & 3 & 2 & 6 & 8 & 9 & 5 & 5 & 7 \\
\hline & $\operatorname{MAPD}_{1}(\%)$ & 14 & 5 & 3 & 12 & 11 & 18 & 10 & 8 & 11 \\
\hline & $\mathrm{RMSE}_{2}(\mathrm{~m})$ & 8 & 2 & 2 & 4 & 6 & 6 & 5 & 5 & 6 \\
\hline & $\operatorname{MAPD}_{2}(\%)$ & 19 & 4 & 2 & 5 & 11 & 13 & 5 & 8 & 9 \\
\hline & $\mathrm{N}$ & 48 & 48 & 48 & 48 & 48 & 48 & 47 & 44 & 48 \\
\hline \multirow{10}{*}{ Stepwise } & A & -33 & -22 & 2 & -35 & -21 & -13 & -9 & 7 & -5 \\
\hline & B & 1.17 & 1.07 & 1.00 & 1.17 & 0.94 & 0.66 & 0.99 & 0.89 & 0.99 \\
\hline & $\sigma_{\mathrm{A}}$ & 19 & 19 & 1 & 19 & 16 & 10 & 22 & 1 & 23 \\
\hline & $\sigma_{\mathrm{B}}$ & 0.32 & 0.31 & 0.01 & 0.32 & 0.26 & 0.17 & 0.36 & 0.02 & 0.37 \\
\hline & $\gamma$ & 0.73 & 0.70 & 1.00 & 0.73 & 0.73 & 0.78 & 0.48 & 1.0 & 0.39 \\
\hline & $\operatorname{RMSE}_{1}(\mathrm{~m})$ & 28 & 23 & 2 & 30 & 28 & 34 & 22 & 3 & 22 \\
\hline & $\operatorname{MAPD}_{1}(\%)$ & 46 & 32 & 3 & 49 & 45 & 58 & 14 & 5 & 21 \\
\hline & $\mathrm{RMSE}_{2}(\mathrm{~m})$ & 16 & 16 & 1 & 16 & 13 & 8 & 19 & 1 & 21 \\
\hline & $\operatorname{MAPD}_{2}(\%)$ & 55 & 40 & 1 & 61 & 41 & 30 & 49 & 2 & 50 \\
\hline & $\mathrm{N}$ & 6 & 6 & 6 & 6 & 6 & 6 & 6 & 4 & 6 \\
\hline \multirow{10}{*}{$\begin{array}{c}\text { Classical + } \\
\text { Stepwise }\end{array}$} & A & -8 & -4 & 1 & -7 & -5 & -9 & -3 & -1 & 2 \\
\hline & B & 0.98 & 1.01 & 1.00 & 1.02 & 0.95 & 0.98 & 1.01 & 1.03 & 1.01 \\
\hline & $\sigma_{\mathrm{A}}$ & 4 & 3 & 1 & 3 & 3 & 3 & 3 & 2 & 3 \\
\hline & $\sigma_{B}$ & 0.07 & 0.05 & 0.01 & 0.06 & 0.06 & 0.06 & 0.05 & 0.03 & 0.06 \\
\hline & $\gamma$ & 0.87 & 0.94 & 0.92 & 0.91 & 0.90 & 0.88 & 0.93 & 0.97 & 0.90 \\
\hline & $\operatorname{RMSE}_{1}(\mathrm{~m})$ & 14 & 8 & 2 & 11 & 12 & 14 & 9 & 5 & 10 \\
\hline & $\operatorname{MAPD}_{1}(\%)$ & 18 & 8 & 3 & 16 & 15 & 22 & 10 & 8 & 12 \\
\hline & $\mathrm{RMSE}_{2}(\mathrm{~m})$ & 11 & 7 & 2 & 9 & 9 & 10 & 8 & 5 & 10 \\
\hline & $\mathrm{MAPD}_{2}(\%)$ & 29 & 12 & 2 & 18 & 20 & 27 & 9 & 7 & 12 \\
\hline & $\mathrm{N}$ & 54 & 54 & 54 & 54 & 54 & 54 & 53 & 48 & 54 \\
\hline
\end{tabular}

Note: $A$-Intercept $; B-$ Slope $; \sigma_{A}-$ Standard deviation in intercept; $\sigma_{B}-$ Standard deviation in slope $; \gamma-$ Correlation coefficient; $R M S E_{1}-$ Root mean square error relative to 1:1 relationship; $M A P D_{1}-$ Mean absolute percent deviation relative to 1:1 relationship; $R M S E_{2}-$ Root mean square error relative to best fit line; $\mathrm{MAPD}_{2}-$ Mean absolute percent deviation relative to best fit line; $N-$ Number of data points. * Method failed to locate MLD in 1 or more cruises. Table only includes statistics of successful MLD estimated cases. 